\title{
Antimicrobial susceptibility and genetic characteristics of Neisseria gonorrhoeae isolates from Vietnam, 2011
}

Birgitta Olsen ${ }^{1 \dagger}$, Pham Thi Lan ${ }^{2 \dagger}$, Daniel Golparian ${ }^{3}$, Emma Johansson $^{3}$, Tran Hau Khang ${ }^{2}$ and Magnus Unemo ${ }^{3^{*}}$

\begin{abstract}
Background: Antimicrobial resistance (AMR) in Neisseria gonorrhoeae is a major public health concern worldwide. In Vietnam, knowledge regarding N. gonorrhoeae prevalence and AMR is limited, and data concerning genetic characteristics of $\mathrm{N}$. gonorrhoeae is totally lacking. Herein, we investigated the phenotypic AMR (previous, current and possible future treatment options), genetic resistance determinants for extended-spectrum cephalosporins (ESCS), and genotypic distribution of N. gonorrhoeae isolated in 2011 in Hanoi, Vietnam.

Methods: N. gonorrhoeae isolates from Hanoi, Vietnam isolated in $2011(n=108)$ were examined using antibiograms (Etest for 10 antimicrobials), Neisseria gonorrhoeae multi-antigen sequence typing (NG-MAST), and sequencing of ESC resistance determinants (penA, $m$ trR and penB).

Results: The levels of in vitro resistance were as follows: ciprofloxacin $98 \%$, tetracycline $82 \%$, penicillin G 48\%, azithromycin $11 \%$, ceftriaxone $5 \%$, cefixime $1 \%$, and spectinomycin $0 \%$. The MICs of gentamicin $(0.023-6 \mathrm{mg} / \mathrm{L})$, ertapenem (0.002-0.125 mg/L) and solithromycin ( $<0.016-0.25 \mathrm{mg} / \mathrm{L})$ were relatively low. No penA mosaic alleles were found, however, 78\% of the isolates contained an alteration of amino acid A501 (A501V (44\%) and A501T (34\%)) in the encoded penicillin-binding protein 2. A single nucleotide (A) deletion in the inverted repeat of the promoter region of the $m t r R$ gene and amino acid alterations in MtrR was observed in 91\% and $94 \%$ of the isolates, respectively. penB resistance determinants were detected in $87 \%$ of the isolates. Seventy-five different NG-MAST STs were identified, of which 59 STs have not been previously described.

Conclusions: In Vietnam, the highly diversified gonococcal population displayed high in vitro resistance to antimicrobials previously recommended for gonorrhoea treatment (with exception of spectinomycin), but resistance also to the currently recommended ESCs were found. Nevertheless, the MICs of three potential future treatment options were low. It is essential to strengthen the diagnostics, case reporting, and epidemiologic surveillance of gonorrhoea in Vietnam. Furthermore, the surveillance of gonococcal AMR and gonorrhoea treatment failures is imperative to reinforce. Research regarding novel antimicrobial treatment strategies (e.g., combination therapy) and new antimicrobials is crucial for future treatment of gonorrhoea.
\end{abstract}

Keywords: Neisseria gonorrhoeae, Gonorrhoea, Vietnam, Antimicrobial resistance, Extended-spectrum cephalosporins (ESCs), Ceftriaxone, Cefixime, Resistance determinants, penA, NG-MAST

\footnotetext{
* Correspondence: magnus.unemo@orebroll.se

${ }^{\dagger}$ Equal contributors

${ }^{3}$ WHO Collaborating Centre for Gonorrhoea and Other STIs, National

Reference Laboratory for Pathogenic Neisseria, Department of Laboratory

Medicine, Clinical Microbiology, Örebro University Hospital, Örebro SE-701 85,

Sweden

Full list of author information is available at the end of the article
}

\section{Ciomed Central}

(c) 2013 Olsen et al.; licensee BioMed Central Ltd. This is an Open Access article distributed under the terms of the Creative Commons Attribution License (http://creativecommons.org/licenses/by/2.0), which permits unrestricted use, distribution, and reproduction in any medium, provided the original work is properly cited. 


\section{Background}

Infections caused by Neisseria gonorrhoeae are major public health problems globally. In 2008, the World Health Organization (WHO) estimated 106 million new cases of gonorrhoea among adults worldwide. This places gonorrhoea as the most common bacterial sexually transmitted infection (STI), that is, together with Chlamydia trachomatis infections also estimated to 106 million cases. The highest gonorrhoea incidence was in the WHO Western Pacific region (WPR), estimated to 42 million cases [1]. In general, the highest rates of gonorrhoea have been found in developing countries and especially in lower socioeconomic groups, men who have sex with men (MSM), commercial sex workers (CSWs) and their clients [1,2].

$N$. gonorrhoeae has developed resistance to all antimicrobials previously recommended as first-line treatment of gonorrhoea, e.g. penicillins, tetracyclines and fluoroquinolones, as well as macrolides such as erythromycin and azithromycin. Extended-spectrum cephalosporins (ESCs) are currently the recommended first-line antimicrobials in most countries worldwide. However, recent two decades in vitro resistance also to ESCs have emerged and spread [2-6]. Verified treatment failures with the oral ESC cefixime have been reported in Japan and recently in several European countries [6-12]. With the injectable ESC ceftriaxone, a few cases of confirmed treatment failure of pharyngeal gonorrhoea have been reported [13-16] and most worryingly the first three extensively-drug resistant (XDR) [5] gonococcal strains with high-level resistance to ceftriaxone have been described $[9,16,17]$. Ceftriaxone is also the last remaining option for empirical first-line antimicrobial monotherapy of gonorrhoea. In this developing situation, including the fear that gonorrhoea may become untreatable, the WHO [18], European Centre for Disease Prevention and Control (ECDC) [19] and Centers for Disease Control and Prevention (CDC), USA [20] have published action/response plans to combat and mitigate the widespread of multidrug-resistant gonorrhoea. However, even if these action/response plans will be fully implemented ultimately it is vital to develop new treatment strategies and particularly novel antimicrobials. Gentamicin, ertapenem and solithromycin have been previously investigated and may be effective treatment alternatives, in antimicrobial monotherapy and particularly in combination therapy [6,21-25].

Mutations in the penA gene encoding the penicillinbinding protein 2 (PBP2) is the main determinant for decreased susceptibility and resistance to ESCs. Acquisition of a penA mosaic gene or an alteration of amino acid A501 in PBP2 result in a lower affinity for ESCs and consequently a decreased ESC susceptibility [6,9,16,26-32]. Mutations in the promoter or coding sequence of the repressor gene $m t r R$ cause over-expression of the MtrCDE efflux pump system that export the ESCs out from the cell. This further decreases the susceptibility to ESCs [6,9,16,28,32-35]. Alterations of amino acid G101 and A102 in the porin PorB1b (the penB resistance determinant), which is encoded by the porB1b gene, result in decreased permeability and further decreased susceptibility to ESCs $[6,9,16,28,32,34,36,37]$. There is still at least one unknown non-transformable ESC resistance determinant [6,9,16,32].

Historically, antimicrobial resistance (AMR) in N. gonorrhoeae appears to mostly have emerged in WHO WPR and subsequently spread globally, by sex tourists and travellers [2,4-6]. In WHO WPR, Vietnam is the easternmost country on the Indochina Peninsula in Southeast Asia; bordering to China, Laos, and Cambodia and with an estimated population of about 90 millions. In Vietnam, for treatment of gonorrhoea ceftriaxone $250 \mathrm{mg} \times 1$ intramuscularly is the recommended first-line and cefixime $400 \mathrm{mg} \times 1$ orally the recommended second-line. However, a wide variety of antimicrobials such as penicillins, fluoroquinolones, macrolides, and spectinomycin may still be used for treatment. Worryingly, the knowledge regarding $N$. gonorrhoeae AMR in Vietnam is limited, and data concerning genetic characteristics (molecular epidemiology and genetic resistance determinants) is totally lacking. Regarding prevalence of gonorrhoea in Vietnam, some few studies have showed low prevalences among women of reproductive age [38,39], one study of female sex workers described a prevalence of $14.9 \%$ [40] and one study of men who have sex with men reported a prevalence of $1.8 \%$ for gonorrhoea and $4.7 \%$ for gonorrhoea/chlamydia [41]. In general, the gonorrhoea diagnostics in Vietnam is suboptimal and individuals commonly prefer private healthcare providers, self-medication or treatment by drug sellers instead of accessing public services $[38,42]$. Accordingly, no reliable national incidence data in regard to bacterial STIs, including gonorrhoea, exist because the STI diagnostics (quality including availability), case reporting (particularly among private health care providers) and epidemiological surveillance are suboptimal.

The aims of the present study were to investigate the phenotypic AMR (previous, current and possible future treatment options), genetic ESC resistance determinants, and genotypic distribution of $N$. gonorrhoeae isolated in 2011 in Hanoi, Vietnam.

\section{Methods}

\section{Neisseria gonorrhoeae isolates}

All $108 N$. gonorrhoeae isolates were obtained at the National Hospital of Dermatology and Venerology, Hanoi, Vietnam from January to September 2011. The isolates were cultured from consecutive symptomatic gonorrhoea patients; 19 females, 85 males, and four patients lacking data of sex and age. Mean age for the females was 28.5 years (median age: 26.5 years; range: 6 to 40 years) and for the males 29 years (median age: 27 years; range: 18 to 60 years). Of the patients, $72 \%$ were from Hanoi, $18 \%$ from other Red 
river delta provinces, $6 \%$ from different other provinces, and for $4 \%$ no information regarding place of accommodation was available.

All isolates were lyophilized and sent to the WHO Collaborating Centre for Gonorrhoea and Other STIs, Örebro University Hospital, Sweden, for species confirmation by culture on selective agar media, a sugar utilization test and the PhadeBact GC Monoclonal test (Bactus AB, Solna, Sweden), and subsequently preserved as previously described [43]. All examined gonococcal isolates were cultured and stored as part of the routine diagnostics (standard care) and no patient identification information was used in the study.

\section{Antimicrobial susceptibility testing}

The minimum inhibitory concentration (MIC; $\mathrm{mg} / \mathrm{L}$ ) of cefixime, ceftriaxone, penicillin G, azithromycin, ciprofloxacin, spectinomycin, tetracycline, gentamicin, and ertapenem were analysed using the Etest method (bioMerieux $A B$, Solna, Sweden), according to the instructions from the manufacturer, and to solithromycin with agar dilution method, in accordance with the Clinical and Laboratory Standards Institute (CLSI (M100-S22)). All results were interpreted using whole MIC dilutions and where available, breakpoints for susceptibility (S) and resistance (R) according to The European Committee on Antimicrobial Susceptibility Testing (EUCAST [www.eucast.org]) were used (Table 1). For gentamicin, ertapenem and solithromycin, no breakpoints are stated by any organization.

\section{Isolation of genomic DNA}

Bacterial DNA was isolated in the robotized NorDiag Bullet instrument (NorDiag ASA Company, Oslo, Norway) using the BUGS'n BEADS ${ }^{\mathrm{TM}}$ STI-fast kit (NorDiag ASA
Company, Oslo, Norway), according to the instructions from the manufacturer.

\section{Molecular epidemiological typing}

N. gonorrhoeae multiantigen sequence typing (NG-MAST) was performed as previously described $[44,45]$. NG-MAST allele numbers of the more variable segments of porB and $t b p B$, and sequence types (STs) were assigned using the NG-MAST website (www.ng-mast.net).

\section{Sequencing of genetic ESC resistance determinants}

Detection of ESC resistance determinants in $N$. gonorrhoeae, i.e. penA mosaic alleles, alterations of A501 in $\mathrm{PBP} 2$, mutations in the promoter and/or coding sequence of the $m t r R$ gene, and the penB resistance determinant, were performed by sequencing as previously described $[34,45]$.

\section{Sequence alignments}

Multiple-sequence alignments of nucleotide sequences and the deduced corresponding amino acid sequences were performed in the software BioEdit Sequence Alignment Editor version 7.0.9.0 with manual adjustment.

\section{Results}

\section{Antimicrobial susceptibility}

The antimicrobial susceptibility of all isolates are summarised in Table 1.

Briefly, the levels of in vitro resistance were as follows: ciprofloxacin $98 \%$, tetracycline $82 \%$, penicillin G $48 \%$, azithromycin $11 \%$, ceftriaxone $5 \%$, cefixime $1 \%$, and spectinomycin 0\%. For gentamicin (MIC range: $0.023-6 \mathrm{mg} / \mathrm{L}$ ), ertapenem $(0.002-0.125 \mathrm{mg} / \mathrm{L})$ and solithromycin $(<0.016-$ $0.25 \mathrm{mg} / \mathrm{L}$ ) there are no breakpoints stated by any

Table 1 Antimicrobial susceptibility of 108 Neisseria gonorrhoeae isolates from Hanoi, Vietnam in 2011

\begin{tabular}{|c|c|c|c|}
\hline Antimicrobial (Breakpoints (mg/L)) & Susceptible no. (\%) & Intermediate no. (\%) & Resistant no. (\%) \\
\hline Ciprofloxacin $(S \leq 0.032, I=0.064, R>0.064)^{*}$ & $2(2)$ & 0 & $106(98)$ \\
\hline Tetracycline $(S \leq 0.5, I=1.0, R>1.0)^{*}$ & $7(6)$ & $13(12)$ & $88(82)$ \\
\hline Penicillin G $(S \leq 0.064, \mid=0.125-1.0, R>1.0)^{*}$ & $2(2)$ & $54(50)$ & $52(48)$ \\
\hline Azithromycin $(S \leq 0.25, I=0.5, R>0.5)^{*}$ & $67(62)$ & $29(27)$ & $12(11)$ \\
\hline Ceftriaxone $(S \leq 0.125, I=N A, R>0.125)^{*}$ & $103(95)$ & NA & $5(5)$ \\
\hline Cefixime $(S \leq 0.125, I=N A, R>0.125)^{*}$ & $107(99)$ & NA & $1(1)$ \\
\hline \multirow[t]{2}{*}{ Spectinomycin $(S \leq 64, I=N A, R>64)^{*}$} & $108(100)$ & NA & 0 \\
\hline & MIC range (mg/L) & $\mathrm{MIC}_{50}(\mathrm{mg} / \mathrm{L})$ & $\mathrm{MIC}_{90}(\mathrm{mg} / \mathrm{L})$ \\
\hline Gentamicin** & $0.032-8$ & 4 & 4 \\
\hline Ertapenem $* *$ & $0.002-0.125$ & 0.012 & 0.032 \\
\hline Solithromycin ${ }^{* *}$ & $<0.016-0.25$ & 0.064 & 0.125 \\
\hline
\end{tabular}

NA, not applicable.

* Breakpoints for susceptible ( $\leq x \mathrm{mg} / \mathrm{L})$ and resistant $(\mathrm{R}>\mathrm{y} \mathrm{mg} / \mathrm{L})$ according to The European Committee on Antimicrobial Susceptibility Testing (EUCAST; www. eucast.org).

** Breakpoints not stated by any organization. 
organization, however, the MICs were relatively low, indicating a susceptible gonococcal population (Table 1).

According to the breakpoints stated by the EUCAST, five (5\%) isolates displayed in vitro resistance to ceftriaxone (all having MIC $=0.25 \mathrm{mg} / \mathrm{L}$ ) and $1(1 \%)$ to cefixime $(\mathrm{MIC}=0.25 \mathrm{mg} / \mathrm{L})$. However, in Japan also gonococcal isolates with cefixime MIC of $0.125 \mathrm{mg} / \mathrm{L}$ have resulted in treatment failure with cefixime $(200 \mathrm{mg} \times 2)$ [7]. Accordingly, isolates with an ESC MIC of $0.125 \mathrm{mg} / \mathrm{L}$ might be considered as having at least an in vitro decreased susceptibility to ESCs. In the present study, the number of isolates with an ESC MIC $\geq 0.125 \mathrm{mg} / \mathrm{L}$ were high, i.e., $30(28 \%)$ and $9(8 \%)$ for ceftriaxone and cefixime, respectively.

ESC genetic resistance determinants (pen $A, m t r R$ and pen $B$ ) The NG-MAST STs, presence of ESC resistance determinants, and MICs of ESCs among all the isolates with in vitro resistance or decreased susceptibility to ESCs $(\mathrm{MIC} \geq 0.125 \mathrm{mg} / \mathrm{L} ; \mathrm{n}=30)$ are summarized in Table 2 .

None of the 30 isolates with in vitro resistance or decreased susceptibility (MIC $\geq 0.125 \mathrm{mg} / \mathrm{L}$ ) to ceftriaxone $(\mathrm{n}=30)$ and cefixime $(\mathrm{n}=9)$ contained a penA mosaic allele. However, 17 (57\%) of those 30 isolates contained an A501T alteration and $12(40 \%)$ had an A501V alteration in PBP2, and all 30 isolates contained $m$ trR and penB resistance determinants (Table 2).

Moreover, in the entire material (108 isolates) no isolates containing a penA mosaic allele were found. However, 47 (44\%) of the isolates contained a PBP2 A501V alteration and 37 (34\%) a PBP2 A501T alteration. The alleles, according to the previously published numbering of PBP2 amino acid sequences in $N$. gonorrhoeae [16], were PBP allele XVIII $(n=37)$, A501V New allele $(n=28)$, XIII $(n=13)$, XXI $(n=3)$, XXXIII $(n=2)$, and A501V New allele $(n=1)$. The MICs of cefixime and ceftriaxone for the isolates with PBP2 A501 alterations ranged from $<0.016-0.25 \mathrm{mg} / \mathrm{L}$ (mean MIC: $0.064 \mathrm{mg} / \mathrm{L}$ ) and $0.016-0.25 \mathrm{mg} / \mathrm{L}$ (mean MIC: $0.064 \mathrm{mg} / \mathrm{L}$ ), respectively.

A single nucleotide (A) deletion in the inverted repeat of the promoter region of the $m t r R$ gene was observed in 98 (91\%) of the 108 isolates. Amino acid alterations in MtrR were found in 102 (94\%) isolates. Accordingly, 45 (42\%) had alterations in amino acid residue H105, 30 (28\%) in A39, and 27 (25\%) in G45. Furthermore, the recently described C-to-T transition $120 \mathrm{bp}$ upstream of the $m t r C$ start codon (termed mtr120) that results in a consensus -10 element, a novel promoter for $m \operatorname{tr} C D E$ transcription and accordingly increased expression of the MtrC-MtrD-MtrE efflux pump was found in four (4\%) isolates [35].

Alterations in amino acid residues G101 and/or A102 of PorB1b (penB resistance determinant) were detected in $94(87 \%)$ of the isolates. All those isolates possessed an altered G101, and 84 (78\%) contained an additional alteration of A102.

\section{Molecular epidemiological characterisation}

The 108 isolates were assigned to 75 different NG-MAST STs. Fifty-nine (79\%) of these STs have not been previously described. The most prevalent ST was ST4787 $(\mathrm{n}=11)$, followed by ST7720 $(n=5)$, ST7741 $(n=4)$, ST7724 $(n=3)$, and ST7155 $(n=3)$. Of those STs, all except ST4787 were

Table 2 Neisseria gonorrhoeae with decreased susceptibility or resistance to cefixime or ceftriaxone $(\geq 0.125)$ isolated in Hanoi, Vietnam in $2011(n=30)$

\begin{tabular}{|c|c|c|c|c|c|c|c|}
\hline \multirow[t]{2}{*}{ NG-MAST } & \multicolumn{2}{|c|}{$\begin{array}{l}\text { Minimum inhibitory } \\
\text { concentration }\end{array}$} & \multirow{2}{*}{$\begin{array}{l}\text { penA alteration } \\
\text { A501 alteration } \\
\text { (penA allele [16]) }\end{array}$} & \multicolumn{2}{|c|}{$m t r R$ alteration } & \multicolumn{2}{|c|}{$\begin{array}{l}\text { PorB1b (penB } \\
\text { alteration) }\end{array}$} \\
\hline & Cefixime & Ceftriaxone & & A-deletion in promoter region & MtrR coding region & G101 & A102 \\
\hline ST4787 $(n=1)$ & 0.25 & 0.25 & $\mathrm{~T}(\mathrm{XV} \mid \mathrm{II})$ & Yes & А39T & K & $\mathrm{D}$ \\
\hline ST4787 $(n=2)$ & 0.125 & 0.25 & $\mathrm{~T}(\mathrm{XVIII)}$ & Yes & A39T & K & $\mathrm{D}$ \\
\hline ST4787 $(n=1)$ & 0.125 & 0.125 & V (A501 New**) & Yes & H105Y & K & D \\
\hline ST4787 $(n=3)$ & 0.064 & 0.125 & $\mathrm{~T}(\mathrm{XV} I I I)$ & Yes & A39T & K & D \\
\hline ST4787 $(n=1)$ & 0.032 & 0.125 & $\mathrm{~T}(\mathrm{XVIII)}$ & Yes & A39T & K & D \\
\hline ST7159 $(n=2)$ & 0.064 & $0.125 / 0.25$ & $\mathrm{~T}(\mathrm{XVIII})$ & Yes & D79N T86A H105Y & K & $D$ \\
\hline ST4676 $(n=1)$ & 0.125 & 0.25 & $\mathrm{~T}(\mathrm{XVIII})$ & Yes & H105Y & K & G \\
\hline $3 \mathrm{STS}^{\mathrm{a}}(\mathrm{n}=4)$ & 0.125 & 0.125 & $\mathrm{~T}(\mathrm{XV} I I I)$ & Yes & А39Т & $K / D^{b}$ & $\mathrm{D} / \mathrm{G}^{\mathrm{b}}$ \\
\hline 12 STs $(n=15)$ & 0.064 & 0.125 & $\mathrm{TN}^{\mathrm{T}} \mathrm{T}^{\mathrm{C}}$ & Yes & A39T G45D H105Yd & $\mathrm{K} / \mathrm{D} / \mathrm{T}^{\mathrm{e}}$ & $\mathrm{D} / \mathrm{G} / \mathrm{WT}^{\mathrm{e}}$ \\
\hline
\end{tabular}

NG-MAST, Neisseria gonorrhoeae multiantigen sequence typing; ST, sequence type.

a ST7739 $(n=2)$, ST7160 $(n=1)$ and ST7741 $(n=1)$

${ }^{b}$ G101D/A102G $(n=2)$ and G101K/A102D $(n=1)$.

${ }^{c}$ A501V New** $(n=9)$, XVIII $(n=3)$, XIII $(n=2)$ and XII $(n=1)$.

${ }^{d}$ H105Y $(n=8)$, A39T $(n=4)$, and G45D $(n=3)$.

e G101K/A102D (n=11), G101D/A102G ( $n=2)$, G101D/WT $(n=1)$, G101T/A102D $(n=1)$. 
novel STs. Twelve STs contained two isolates and 58 STs were represented by single isolates (Table 3 ).

Eight $(73 \%)$ of the ST4787 isolates $(\mathrm{n}=11)$ displayed in vitro resistance or decreased susceptibility to ceftriaxone $(\mathrm{MIC} \geq 0.125 \mathrm{mg} / \mathrm{L})$ and four (36\%) of them resistance or decreased susceptibility to cefixime (MIC $\geq 0.125 \mathrm{mg} / \mathrm{L}$ ). Ten (91\%) of the ST4787 isolates contained an identical A501T altered PBP2 allele (XVIII), a single nucleotide (A) deletion in the $m t r R$ promoter, A39T alteration in MtrR and penB (G101K/A102D) (Table 2).

\section{Discussion}

In the present study, the susceptibility to previous, current and possible future antimicrobial treatment options in $N$. gonorrhoeae isolated in 2011 in Vietnam were studied. Exceedingly high prevalence of resistance was observed for previous first-line antimicrobials such as ciprofloxacin (98\%), tetracycline (82\%) and penicillin G (48\%), but also relatively high for azithromycin (11\%) (Table 1$)$. This is in accordance with previous studies from other countries in WHO WPR such as Japan, The Philippines, China, Hong Kong, Korea and Taiwan [4,46-51], South Asia, e.g., India, Pakistan, Thailand, Sri Lanka and Bhutan [47,52,53], and many other regions globally [3-6,18,19,54-58]. None of these antimicrobials can be recommended for first-line empiric therapy of gonorrhoea in Vietnam as well as in most parts of the world.

Most worryingly, $5 \%$ of the gonococcal isolates in Vietnam displayed in vitro resistance to ceftriaxone and $1 \%$ to cefixime (Table 1). Furthermore, in total 30 (28\%) and $9(8 \%)$ displayed in vitro resistance or decreased susceptibility (MIC $\geq 0.125 \mathrm{mg} / \mathrm{L}$ ) to ceftriaxone and cefixime, respectively (Table 2). All except one of these 30 isolates contained an A501 alteration in PBP2, and all 30 comprised $m t r R$ and penB resistance determinants. All these resistance determinants have previously been reported as associated with decreased susceptibility and resistance to ESCs [3,5,6,9-11,14-17,25-32,34,51,58,59]. However, in Vietnam no isolates with a mosaic PBP allele, which has been strongly associated with decreased susceptibility and resistance to ESCs in many countries, was found $[3,5,6,31,32,58]$. The predominance of A501altered penA alleles in a gonococcal population with in vitro resistance and decreased susceptibility to ESCs as observed in Vietnam has also been previously described in publications from other countries such as Korea [28] and Australia $[59,60]$. In the present study, the MICs of cefixime and ceftriaxone for the isolates with PBP2 A501 alterations ranged from $<0.016-0.25 \mathrm{mg} / \mathrm{L}$ and $0.016-0.25 \mathrm{mg} / \mathrm{L}$, respectively. This large variety in the MICs of ESCs among these isolates could not be explained by the presence or absence of other resistance determinants such as $m t r R$ and penB. The reasons for the highly variable ESC MICs of isolates with A501-altered PBP2 remain unknown, but
Table 3 Minimum inhibitory concentrations (MICs, mg/L) of cefixime and ceftriaxone for Neisseria gonorrhoeae $(n=108)$ isolated in Hanoi, Vietnam in 2011

\begin{tabular}{|c|c|c|c|c|c|c|}
\hline \multirow{2}{*}{$\begin{array}{l}\text { NG-MAST ST } \\
\text { (No. of isolates) }\end{array}$} & \multirow[t]{2}{*}{ Anti-microbial } & \multicolumn{5}{|c|}{ No. of isolates with MIC (mg/L): } \\
\hline & & $\leq 0.016$ & 0.032 & 0.064 & 0.125 & 0.25 \\
\hline \multirow[t]{2}{*}{$4787(11)$} & IX & & 3 & 4 & 3 & 1 \\
\hline & $\mathrm{TX}$ & & & 3 & 5 & 3 \\
\hline \multirow[t]{2}{*}{$7720(5)^{a}$} & IX & 1 & 4 & & & \\
\hline & TX & & 5 & & & \\
\hline \multirow[t]{2}{*}{$7741(4)^{a}$} & IX & & & 3 & 1 & \\
\hline & $\mathrm{TX}$ & & & & 4 & \\
\hline \multirow[t]{2}{*}{$7155(3)^{a}$} & IX & & 1 & 2 & & \\
\hline & $\mathrm{TX}$ & & 2 & 1 & & \\
\hline \multirow[t]{2}{*}{$7724(3)^{a}$} & IX & & 3 & & & \\
\hline & $\mathrm{TX}$ & & & 3 & & \\
\hline \multirow[t]{2}{*}{$3493(2)$} & IX & 2 & & & & \\
\hline & $\mathrm{TX}$ & 2 & & & & \\
\hline \multirow[t]{2}{*}{$7139(2)^{a}$} & IX & 2 & & & & \\
\hline & $\mathrm{TX}$ & 2 & & & & \\
\hline \multirow[t]{2}{*}{$7140(2)^{a}$} & IX & & 1 & 1 & & \\
\hline & TX & & 2 & & & \\
\hline \multirow[t]{2}{*}{$7159(2)^{a}$} & IX & & & 2 & & \\
\hline & TX & & & & 1 & 1 \\
\hline \multirow[t]{2}{*}{$7718(2)^{a}$} & IX & & & 2 & & \\
\hline & $\mathrm{TX}$ & & & & 2 & \\
\hline \multirow[t]{2}{*}{$7722(2)^{a}$} & IX & 2 & & & & \\
\hline & TX & & 2 & & & \\
\hline \multirow[t]{2}{*}{$7731(2)^{a}$} & IX & & 2 & & & \\
\hline & TX & & 2 & & & \\
\hline \multirow[t]{2}{*}{$7734(2)^{a}$} & IX & & & 2 & & \\
\hline & $\mathrm{TX}$ & & & 1 & 1 & \\
\hline \multirow[t]{2}{*}{$7735(2)^{a}$} & IX & & 2 & & & \\
\hline & $\mathrm{TX}$ & & 1 & 1 & & \\
\hline \multirow[t]{2}{*}{$7736(2)^{a}$} & IX & & 1 & 1 & & \\
\hline & TX & & & 2 & & \\
\hline \multirow[t]{2}{*}{$7739(2)^{a}$} & IX & & & & 2 & \\
\hline & $\mathrm{TX}$ & & & & 2 & \\
\hline \multirow[t]{2}{*}{$7748(2)^{a}$} & IX & & 1 & 1 & & \\
\hline & TX & & 1 & 1 & & \\
\hline \multirow[t]{2}{*}{ Unique STs $(58)^{b}$} & IX & 15 & 16 & 25 & 2 & \\
\hline & $\mathrm{TX}$ & 12 & 15 & 20 & 10 & 1 \\
\hline
\end{tabular}

NG-MAST, Neisseria gonorrhoeae multiantigen sequence typing; ST, sequence type;

MIC, minimum inhibitory concentration; IX, cefixime; TX, ceftriaxone. ${ }^{a}$ New sequence types found in the present study.

${ }^{b}$ Unique STs represented by single isolates. 
possibly the isolates with higher ESC MICs also contain the unknown non-transformable penicillin and cephalosporin resistance determinant "factor X" [6,9,16,32]. Accordingly, detection of the currently known ESC genetic resistance determinants (penA mosaic allele or alteration of A501 in PBP2, $m$ trR and penB) does not strictly reflect the exact MICs of ESCs and, accordingly, cannot replace traditional culture-based AMR testing (which needs to be strengthened worldwide) or be used in the management of clinical patients. However, detection of genetic resistance determinants combined with molecular epidemiological typing (NG-MAST) can still be valuable for surveillance purposes, i.e. to monitor the emergence and spread of isolates with decreased susceptibility or resistance to ESCs, enhancing our knowledge regarding the effects on ESC MICs of different penA alleles, etc. The present study shows that the prevalence of gonococcal isolates with phenotypic and genetic resistance or decreased susceptibility to ESCs in Vietnam is high. Longitudinal studies in Vietnam examining the ESC MICs over time supplemented by determination of genetic resistance determinants would be exceedingly valuable.

Disquietingly, despite full implementation of the action/response plans recently launched [18-20], ultimately new treatment strategies and particularly novel antimicrobials are essential to develop. In the United Kingdom [61], Europe [62] and USA [63], the recently revised treatment guidelines all recommend dual antimicrobial therapy (mainly with ceftriaxone plus azithromycin). However, in vitro and in vivo resistance to both ceftriaxone and azithromycin have already been verified and dual antimicrobial therapy may not be feasible and/or affordable in all less-resourced settings. Accordingly, new antimicrobials for treatment of gonorrhoea are essential to develop. There are few new compounds in sight $[3,6,16,64]$. The new fluoroketolide solithromycin (class: macrolides) has recently been investigated and showed an activity superior to that of most other antimicrobials previously or currently recommended for treatment of gonorrhoea [23]. In the present study, despite that $11 \%(27 \%)$ of the isolates were resistant (intermediate resistance) to azithromycin the MIC range of solithromycin was only $<0.016-0.25 \mathrm{mg} / \mathrm{L}$ and $\mathrm{MIC}_{90}$ was $0.125 \mathrm{mg} / \mathrm{L}$. Accordingly, these results further support solithromycin as a possible future option for single and particularly dual antimicrobial therapy of gonorrhoea. Ertapenem, a parenteral 1- $\beta$-methyl-carbapenem, has in a previous study shown advantages over ceftriaxone for ceftriaxone-resistant isolates [25]. In the present study, all 30 isolates with in vitro resistance $(\mathrm{n}=5)$ and decreased susceptibility $(n=25)$ to ceftriaxone displayed ertapenem MIC values ranging from $0.016 \mathrm{mg} / \mathrm{L}$ to $0.032 \mathrm{mg} / \mathrm{L}$ (Table 2), and the $\mathrm{MIC}_{90}$ for all isolates was $0.032 \mathrm{mg} / \mathrm{L}$. Finally, the aminoglycoside gentamicin has been used for nearly two decades in Malawi to treat gonorrhoea (mainly in syndromic management together with doxycycline), with a remained high in vitro susceptibility in the gonococcal populations [21,24]. An evaluation of gentamicin in vitro susceptibility of $N$. gonorrhoeae isolates in Europe showed that $95 \%$ of 1366 isolates were distributed within a narrow MIC range of 4-8 $\mathrm{mg} / \mathrm{L}$ [65]. In the present study, the $\mathrm{MIC}$ range was $0.032-6 \mathrm{mg} / \mathrm{L}$ and $\mathrm{MIC}_{90}$ was $4 \mathrm{mg} / \mathrm{L}$. There are yet no international interpretative criteria for MICs of solithromycin, ertapenem or gentamicin. However, in studies from Malawi breakpoints have been suggested, that is, susceptible: $\mathrm{MICs} \leq 4 \mathrm{mg} / \mathrm{L}$, intermediate susceptible: $\mathrm{MIC}=8-16 \mathrm{mg} / \mathrm{L}$ and resistant: $\mathrm{MICs} \geq$ $32 \mathrm{mg} / \mathrm{L}$ [21].

Finally, using NG-MAST the present study showed a diversified population of $N$. gonorrhoeae in Hanoi, Vietnam during 2011, with 75 different NG-MAST STs among the 108 isolates. The high number of unique STs $(n=58)$ and STs that have not been described earlier $(n=59)$ may be associated with suboptimal diagnostics (only random gonorrhoea patients and/or isolates are identified), contact tracing (sexual contacts having the identical ST are not traced) and epidemiological surveillance (sexual transmission chains spreading a single ST are not identified or followed-up), STs evolved locally in Vietnam (STs are not previously described because no NG-MAST studies have previously been performed in the country) or imported from abroad. However, some minor ST clusters caused by clonal spread of, e.g. ST4787 $(n=11)$, ST7720 $(n=5)$ and ST7741 $(n=4)$ were identified which indicate some sexual transmission chains. Of the eleven ST4787 isolates, eight displayed in vitro resistance or decreased susceptibility to ceftriaxone and all eleven isolates showed A501 alteration in PBP2 as well as contained $m t r R$ and $p e n B$ resistance determinants.

\section{Conclusions}

N. gonorrhoeae isolated in Vietnam during 2011 showed a high genetic diversity and high levels of in vitro resistance to antimicrobials previously recommended for gonorrhoea treatment, such as ciprofloxacin, tetracycline, penicillin G and azithromycin. Furthermore, 5\% (28\%) of the isolates were in vitro resistant (had a decreased susceptibility) to ceftriaxone, currently the recommended drug of choice for treatment of gonorrhoea. Nevertheless, no resistance to spectinomycin, which remains available in Vietnam, was found and the MICs of three potential future treatment options were low. Research regarding novel antimicrobial treatment strategies (e.g., combination therapy) and new antimicrobials is crucial for future treatment of gonorrhoea.

Finally, it is of great importance to strengthen the $N$. gonorrhoeae diagnostics, case reporting, and surveillance of epidemiology, AMR as well as gonorrhoea treatment failures in Vietnam. 


\section{Competing interests}

The authors declare that they have no competing interests.

\section{Authors' contributions}

MU and PTL designed the study. BO, DG and EJ performed all the laboratory analysis. All authors analysed and interpreted the data, and were involved in the preparations of the paper. All authors read and approved the final manuscript.

\section{Acknowledgments}

This work was supported by the Örebro County Council Research Committee and the Foundation for Medical Research at Örebro University Hospital, Sweden.

\section{Author details}

${ }^{1}$ School of Health and Medical Sciences, Örebro University, Örebro, Sweden. ${ }^{2}$ Department of Dermato-Venerology, Hanoi Medical University; and National Hospital of Dermatology and Venerology, Hanoi, Vietnam. ${ }^{3} \mathrm{WHO}$ Collaborating Centre for Gonorrhoea and Other STIs, National Reference Laboratory for Pathogenic Neisseria, Department of Laboratory Medicine, Clinical Microbiology, Örebro University Hospital, Örebro SE-701 85, Sweden.

Received: 6 December 2012 Accepted: 17 January 2013

Published: 25 January 2013

\section{References}

1. World Health Organization: Global incidence and prevalence of selected curable sexually transmitted infections - 2008. Geneva: World Health Organization; 2012. Available at: http://www.who.int/reproductivehealth/ publications/rtis/2008_STI_estimates.pdf (Accessed: December 30, 2012).

2. Tapsall J: Antimicrobial resistance in Neisseria gonorrhoeae. WHO/CDS/DRS/ 2001.3:16. Geneva: World Health Organisation (WHO); 2001.

3. Barry PM, Klausner JD: The use of cephalosporins for gonorrhea: the impending problem of resistance. Expert Opin Pharmacother 2009, 10:555-577.

4. Tapsall JW: Implications of current recommendations for third-generation cephalosporin use in the WHO Western Pacific Region following the emergence of multiresistant gonococci. Sex Transm Infect 2009, 85:256-258.

5. Tapsall JW, Ndowa F, Lewis DA, et al: Meeting the public health challenge of multidrug- and extensively drug-resistant Neisseria gonorrhoeae. Expert Rev Anti Infect Ther 2009, 7:821-834.

6. Unemo M, Nicholas RA: Emergence of multi-drug resistant, extensively drug-resistant and untreatable gonorrhea. Future Microbiol 2012, 7:1401-1422.

7. Deguchi T, Yasuda M, Ito S: Management of pharyngeal gonorrhea is crucial to prevent the emergence and spread of antibiotic-resistant Neisseria gonorrhoeae. Antimicrob Agents Chemother 2012, 56:4039-4040. author reply 4041-4032.

8. Ison CA, Hussey J, Sankar KN, et al: Gonorrhoea treatment failures to cefixime and azithromycin in England. Euro Surveill 2011, 16(14):pii=19833.

9. Unemo M, Golparian D, Nicholas R, et al: High-level cefixime- and ceftriaxone-resistant Neisseria gonorrhoeae in France: novel penA mosaic allele in a successful international clone causes treatment failure. Antimicrob Agents Chemother 2012, 56:1273-1280.

10. Unemo M, Golparian D, Stary A, et al: First Neisseria gonorrhoeae strain with resistance to cefixime causing gonorrhoea treatment failure in Austria, 2011. Euro Surveill 2011, 16(43):pii=19998.

11. Unemo M, Golparian D, Syversen G, et al: Two cases of verified clinical failures using internationally recommended first-line cefixime for gonorrhoea treatment, Norway, 2010. Euro Surveill 2010, 15(47):pii=19721.

12. Yokoi S, Deguchi T, Ozawa T, et al: Threat to cefixime treatment for gonorrhea. Emerg Infect Dis 2007, 13:1275-1277.

13. Tapsall J, Read P, Carmody C, et al: Two cases of failed ceftriaxone treatment in pharyngeal gonorrhoea verified by molecular microbiological methods. J Med Microbiol 2009, 58:683-687.

14. Unemo M, Golparian D, Hestner A: Ceftriaxone treatment failure of pharyngeal gonorrhoea verified by international recommendations, Sweden, July 2010. Euro Surveill 2011, 16(6):pii=19792.

15. Unemo M, Golparian D, Potocnik M, et al: Treatment failure of pharyngeal gonorrhoea with internationally recommended first-line ceftriaxone verified in Slovenia, September 2011. Euro Surveill 2012, 17(25):pii=20200.

16. Ohnishi M, Golparian D, Shimuta K, et al: Is Neisseria gonorrhoeae initiating a future era of untreatable gonorrhea?: detailed characterization of the first strain with high-level resistance to ceftriaxone. Antimicrob Agents Chemother 2011, 55:3538-3545.

17. Cámara J, Serra J, Ayats J, et al: Molecular characterization of two high-level ceftriaxone-resistant Neisseria gonorrhoeae isolates detected in Catalonia. Spain. J Antimicrob Chemother 2012, 67:1858-1860.

18. World Health Organization (WHO), Department of Reproductive Health and Research: Global action plan to control the spread and impact of antimicrobial resistance in Neisseria gonorrhoeae. Geneva: WHO; 2012:1-36. Available at: http://www.who.int/reproductivehealth/publications/rtis/ 9789241503501 (Accessed: December 30, 2012).

19. European Centre for Disease Prevention and Control (ECDC): Response plan to control and manage the threat of multidrug-resistant gonorrhoea in Europe. Stockholm: ECDC; 2012:1-23. Available at: http://www.ecdc.europa.eu/en/ publications/Publications/1206-ECDC-MDR-gonorrhoea-response-plan.pdf (Accessed: December 30, 2012)

20. Centers for Disease Control and Prevention (CDC): Cephalosporin-resistant Neisseria gonorrhoeae public health response plan; 2012:1-43. Available at: http:// www.cdc.gov/std/gonorrhea/default.htm (Accessed: December 30, 2012).

21. Brown LB, Krysiak R, Kamanga G, et al: Neisseria gonorrhoeae antimicrobial susceptibility in Lilongwe, Malawi, 2007. Sex Transm Dis 2010, 37:169-172.

22. Dowell D, Kirkcaldy RD: Effectiveness of gentamicin for gonorrhoea treatment: systematic review and meta-analysis. Sex Transm Infect 2012, 88:589-594.

23. Golparian D, Fernandes $P$, Ohnishi $M$, et al: In vitro activity of the new fluoroketolide solithromycin (CEM-101) against a large collection of clinical Neisseria gonorrhoeae isolates and international reference strains, including those with high-level antimicrobial resistance: potential treatment option for gonorrhea? Antimicrob Agents Chemother 2012, 56:2739-2742.

24. Ross JD, Lewis DA: Cephalosporin resistant Neisseria gonorrhoeae: time to consider gentamicin? Sex Transm Infect 2012, 88:6-8.

25. Unemo M, Golparian D, Limnios A, et al: In vitro activity of ertapenem versus ceftriaxone against Neisseria gonorrhoeae isolates with highly diverse ceftriaxone MIC values and effects of ceftriaxone resistance determinants: ertapenem for treatment of gonorrhea? Antimicrob Agents Chemother 2012, 56:3603-3609.

26. Ameyama $\mathrm{S}$, Onodera $\mathrm{S}$, Takahata $\mathrm{M}$, et al: Mosaic-like structure of penicillin-binding protein 2 gene (penA) in clinical isolates of Neisseria gonorrhoeae with reduced susceptibility to cefixime. Antimicrob Agents Chemother 2002, 46:3744-3749.

27. Ito M, Deguchi T, Mizutani KS, et al: Emergence and spread of Neisseria gonorrhoeae clinical isolates harboring mosaic-like structure of penicillinbinding protein 2 in central Japan. Antimicrob Agents Chemother 2005, 49:137-143.

28. Lee $\mathrm{SG}$, Lee $\mathrm{H}$, Jeong $\mathrm{SH}$, et al: Various penA mutations together with $m$ trR, porB and ponA mutations in Neisseria gonorrhoeae isolates with reduced susceptibility to cefixime or ceftriaxone. J Antimicrob Chemother 2010, 65:669-675.

29. Osaka K, Takakura T, Narukawa K, et al: Analysis of amino acid sequences of penicillin-binding protein 2 in clinical isolates of Neisseria gonorrhoeae with reduced susceptibility to cefixime and ceftriaxone. J Infect Chemother 2008, 14:195-203.

30. Takahata S, Senju N, Osaki Y, et al: Amino acid substitutions in mosaic penicillin-binding protein 2 associated with reduced susceptibility to cefixime in clinical isolates of Neisseria gonorrhoeae. Antimicrob Agents Chemother 2006, 50:3638-3645.

31. Tomberg J, Unemo M, Davies C, et al: Molecular and structural analysis of mosaic variants of penicillin-binding protein 2 conferring decreased susceptibility to expanded-spectrum cephalosporins in Neisseria gonorrhoeae: role of epistatic mutations. Biochemistry 2010, 49:8062-8070.

32. Zhao S, Duncan M, Tomberg J, et al: Genetics of chromosomally mediated intermediate resistance to ceftriaxone and cefixime in Neisseria gonorrhoeae. Antimicrob Agents Chemother 2009, 53:3744-3751.

33. Folster JP, Johnson PJ, Jackson L, et al: MtrR modulates rpoH expression and levels of antimicrobial resistance in Neisseria gonorrhoeae. J Bacteriol 2009, 19:287-297.

34. Lindberg $R$, Fredlund $H$, Nicholas $R$, et al: Neisseria gonorrhoeae isolates with reduced susceptibility to cefixime and ceftriaxone: association with genetic polymorphisms in penA, $m$ trR, porB1b, and ponA. Antimicrob Agents Chemother 2007, 51:2117-2122.

35. Ohneck EA, Zalucki YM, Johnson PJ, et al: A novel mechanism of high-level, broad-spectrum antibiotic resistance caused by a single base pair change in Neisseria gonorrhoeae. MBio 2011, 2(5):pii=e00187-11. 
36. Olesky M, Hobbs M, Nicholas RA: Identification and analysis of amino acid mutations in porin IB that mediate intermediate-level resistance to penicillin and tetracycline in Neisseria gonorrhoeae. Antimicrob Agents Chemother 2002, 46:2811-2820.

37. Olesky M, Zhao S, Rosenberg RL, et al: Porin-mediated antibiotic resistance in Neisseria gonorrhoeae: ion, solute, and antibiotic permeation through PIB proteins with penB mutations. J Bacterio/ 2006, 188:2300-2308.

38. Lan PT, Lundborg CS, Phuc HD, et al: Reproductive tract infections including sexually transmitted infections: a population-based study of women of reproductive age in a rural district of Vietnam. Sex Transm Infect 2008, 84:126-132.

39. Nguyn MH, Kurtzhals J, Do TT, et al: Reproductive tract infections in women seeking abortion in Vietnam. BMC Womens Health 2009, 9:1.

40. Nguyen TV, Van Khuu N, Le Thi TT, et al: Sexually transmitted infections and risk factors for gonorrhea and chlamydia in female sex workers in Soc Trang. Vietnam. Sex Transm Dis 2008, 35:935-940.

41. Pham QD, Nguyen TV, Hoang CQ, et al: Prevalence of HIV/STIs and associated factors among men who have sex with men in An Giang. Vietnam. Sex Transm Dis 2012, 39:799-806.

42. Lan PT, Faxelid E, Chuc NT, et al: Perceptions and attitudes in relation to reproductive tract infections including sexually transmitted infections in rural Vietnam: a qualitative study. Health Policy 2008, 86:308-317.

43. Unemo M, Olcen $P$, Berglund $T$, et al: Molecular epidemiology of Neisseria gonorrhoeae: sequence analysis of the porB gene confirms presence of two circulating strains. J Clin Microbiol 2002, 40:3741-3749.

44. Martin IM, Ison CA, Aanensen DM, et al: Rapid sequence-based identification of gonococcal transmission clusters in a large metropolitan area. J Infect Dis 2004, 189:1497-1505.

45. Unemo M, Fasth $\mathrm{O}$, Fredlund $\mathrm{H}$, et al: Phenotypic and genetic characterization of the 2008 WHO Neisseria gonorrhoeae reference strain panel intended for global quality assurance and quality control of gonococcal antimicrobial resistance surveillance for public health purposes. J Antimicrob Chemother 2009, 63:1142-1151.

46. Programme AGS: Australian gonococcal surveillance programme annual report, 2010. Commun Dis Intell 2011, 35:229-236.

47. Lahra MM, on behalf of the WHO Western Pacific and South East Asian Gonococcal Antimicrobial Surveillance Programme: Surveillance of antibiotic resistance in Neisseria gonorrhoeae in the WHO Western Pacific and South East Asian Regions, 2010. Commun Dis Intell 2012, 36:95-100.

48. Lee $H$, Hong SG, Soe $Y$, et al: Trends in antimicrobial resistance of Neisseria gonorrhoeae isolated from Korean patients from 2000 to 2006 Sex Transm Dis 2011, 38:1082-1086.

49. Shigemura K, Okada H, Shirakawa T, et al: Susceptibilities of Neisseria gonorrhoeae to fluoroquinolones and other antimicrobial agents in Hyogo and Osaka. Japan. Sex Transm Infect 2004, 80:105-107.

50. Tanaka M, Shimojima M, Saika T, et al: Nationwide antimicrobial susceptibility survey of Neisseria gonorrhoeae isolates in Japan. Kansenshogaku Zasshi 2011, 85:360-365.

51. Huang CT, Yen MY, Wong WW, et al: Characteristics and dissemination of mosaic penicillin-binding protein 2-harboring multidrug-resistant Neisseria gonorrhoeae isolates with reduced cephalosporin susceptibility in northern Taiwan. Antimicrob Agents Chemother 2010, 54:4893-4895.

52. Jabeen K, Nizamuddin S, Irfan S, et al: Increasing trend of resistance to penicillin, tetracycline, and fluoroquinolone resistance in Neisseria gonorrhoeae from Pakistan (1992-2009). J Trop Med 2011, 2011:960501.

53. Sethi S, Golparian D, Bala M, et al: Antimicrobial susceptibility and genetic characteristics of Neisseria gonorrhoeae isolates from India, Pakistan and Bhutan - 2007-2011. BMC Infect Dis 2012, Submitted.

54. Glazkova S, Golparian D, Titov L, et al: Antimicrobial susceptibility/ resistance and molecular epidemiological characteristics of Neisseria gonorrhoeae in 2009 in Belarus. APMIS 2011, 119:537-542.

55. Ison CA: Antimicrobial resistance in sexually transmitted infections in the developed world: implications for rational treatment. Curr Opin Infect Dis 2012, 25:73-78.

56. Kubanova A, Frigo N, Kubanov A, et al: The Russian gonococcal antimicrobial susceptibility programme (RU-GASP)-national resistance prevalence in 2007 and 2008, and trends during 2005-2008. Euro Surveill 2010, 15(14):pii=19533.

57. Martin I, Jayaraman G, Wong T, et al: Trends in antimicrobial resistance in Neisseria gonorrhoeae isolated in Canada: 2000-2009. Sex Transm Dis 2011, 38:892-898.
58. Lewis DA: The gonococcus fights back: is this time a knock out? Sex Transm Infect 2010, 86:415-421.

59. Whiley DM, Goire N, Lambert SB, et al: Reduced susceptibility to ceftriaxone in Neisseria gonorrhoeae is associated with mutations G542S, P551S and P551L in the gonococcal penicillin-binding protein 2. J Antimicrob Chemother 2010, 65:1615-1618.

60. Whiley DM, Limnios EA, Ray S, et al: Diversity of penA alterations and subtypes in Neisseria gonorrhoeae strains from Sydney, Australia, that are less susceptible to ceftriaxone. Antimicrob Agents Chemother 2007, 51:3111-3116

61. Bignell C, Fitzgerald M, BASHH Guideline Development Group: UK national guideline for the management of gonorrhoea in adults, 2011. Int J STD AIDS 2011, 22:541-547.

62. Bignell C, Unemo M, on behalf of the European STI Guidelines Editorial Board: European guideline on the diagnosis and treatment of gonorrhoea in * adults. Int J STD AIDS 2012, In press. Available at: http:// www.iusti.org/regions/Europe/pdf/2012/Gonorrhoea_2012.pdf (Accessed: December 30, 2012)

63. Centers for Disease Control and Prevention (CDC): Update to CDC's Sexually transmitted diseases treatment guidelines, 2010: oral cephalosporins no longer a recommended treatment for gonococcal infections. MMWR Morb Mortal Wkly Rep 2012, 61:590-594.

64. Newman LM, Moran JS, Workowski KA: Update on the management of gonorrhea in adults in the United States. Clin Infect Dis 2007, 44(Suppl 3):S84-101.

65. Chisholm SA, Quaye N, Cole MJ, et al: An evaluation of gentamicin susceptibility of Neisseria gonorrhoeae isolates in Europe. J Antimicrob Chemother 2011, 66:592-595.

\section{doi:10.1186/1471-2334-13-40}

Cite this article as: Olsen et al:: Antimicrobial susceptibility and genetic characteristics of Neisseria gonorrhoeae isolates from Vietnam, 2011. BMC Infectious Diseases 2013 13:40.

\section{Submit your next manuscript to BioMed Central and take full advantage of:}

- Convenient online submission

- Thorough peer review

- No space constraints or color figure charges

- Immediate publication on acceptance

- Inclusion in PubMed, CAS, Scopus and Google Scholar

- Research which is freely available for redistribution

Submit your manuscript at www.biomedcentral.com/submit
C) Biomed Central 